\title{
Visually-impaired children and apps: sharing informal and formal information to guide choice
}

\author{
Sabrina Panesi $^{a}$, Giovanni Paolo Caruso ${ }^{a}$, Jeffrey Earp ${ }^{a}$, Lucia Ferlino ${ }^{a}$, Silvia Dini ${ }^{b}$ \\ ${ }^{a}$ National Research Council of Italy, Institute for Educational Technology, Genoa, Italy, \\ panesi@itd.cnr.it, ferlino@itd.cnr.it, caruso@itd.cnr.it, earp@itd.cnr.it \\ b David Chiossone Onlus Institute, Genoa, Italy, dini@chiossone.it
}

\begin{abstract}
Digital technologies play an important role in the development of partially children, who require early intervention to help sustain and improve their visual capacity throughout childhood, and also to stimulate their other senses so as to prevent any global development deficit.

To this end, therapists, parents and teachers must be able to choose from a wide range of suitable, up-to-date digital resources that have been field tested and are well organized so that they can be located easily and rapidly as required.

To respond to these needs, the authors have been involved in a research project, which has defined the key features of apps that make them suitable for use by and with visually-impaired children. Consequently, the project developed an online environment that combines structured analytical information from experts with the practical know-how of the user community in a joint effort to support the choice of the most suitable resources to use in different contexts and with different users.
\end{abstract}

Keywords: shared knowledge; low-vision; education; rehabilitation; apps.

\section{Low Vision and technology: a brief introduction}

Visually impaired children typically present a lack of visual acuity and reduced visual field, conditions that are often accompanied by low sensitivity to contrast and altered colour perception. Reduced visual space can be like looking through a keyhole, and for this reason the condition is commonly referred to as keyhole vision. In other cases, images can appear washed out, with low contrast levels, or colours may be perceived in a non-standard 
fashion. Generally speaking, visually impaired children have low vision in both eyes both at short and at long range, even when wearing glasses. They are usually classified as being visually impaired when their sight level is only $30 \%$ of that of other children. This means that they are unable to perceive small or even standard text and pictures, but they can make out large images when holding these close up ${ }^{1}$.

In such cases, it is vital that rehabilitation should begin at an early age, drawing on a range of stimuli and support systems, including digital technology. Specifically, tablets and apps, which appeal to children with normal and low vision alike, favour task concentration and the exercising of visual effort (Dini \& Ferlino, 2016). To employ these successfully visually impaired children, rehabilitation therapists, teachers at school and parents at home need to have ready access to apps that meet the specific needs of the young low-vision user.

To be affective, these apps must be age-appropriate and need to have strong appeal to the child so that they can foster motivation and visual effort making. At the same time, they must have certain usability characteristics in terms of interface and graphics.

\section{Towards systematic knowledge sharing}

In education, the need soon arose for tools and services to help locate and, in particular, select suitable information for meeting specific needs. The educational research sector responded by developing cataloguing services to help educators locate and select learning resources. These mostly took the form of databases of material compiled according to an objective and neutral approach emphasizing informativeness (Bonaiuti et al., 2017).

The emergence of Web 2.0 (and subsequently of social networking) had an enormous impact on the education sector as elsewhere. Teachers, parents and other stakeholders continued to consult trusted institutional databases and information services, but at the same time the direct experience of others was also seen as a valuable information source. This is especially true in the special education sector, where the experience of others is taking on particular significance, given that accessibility and usability information is generally absent from institutional learning resource databases.

In the effort to identify and select digital resources suitable for visually impaired children, therapists, teachers and parents are currently engaging in informal, experience-based online communication flows. In this light, researchers in the field of special education have the opportunity to act as pivotal mediators, augmenting and adding value to their established,

1 http://www.who.int/mediacentre/factsheets/fs282/en/ 
well-trusted institutional documentation services with the integration of structured userdriven knowledge sharing initiatives (Pantazopoulos, 2017).

Hence, there are now two pathways available to those seeking out and selecting apps that can be used with, and by, visually impaired children: first, continue consulting institutional documentation services which provide neutral, objective information (formal structured flows); second, consult forums and social networks where users of different types exchange opinions and experiences on apps (uncontrolled spontaneous informal flows).

Clearly, the challenge facing the research sector is to achieve an optimal combination of these two approaches by combining neutral and objective catalogue information about potentially suitable apps with appropriately filtered experiential input from users so as to gain the best of both worlds. The aim of blending these two is to trigger a virtuous circle of effective knowledge sharing.

This challenge has been taken up by a research team located in Genoa, Italy, which for some time has been active in the field of technology-supported educational and social inclusion of people with visual impairment, particularly by offering documentation services dedicated to those ends. In 2016, the team launched a social-network oriented environment called Sharehab ("Share rehabilitation resources"). This combines formal and informal knowledge sharing about apps suitable for use by children and youngsters with visual impairments of various kinds (Figure 1). The platform is substantially aimed at rehab professionals, teachers and parents.

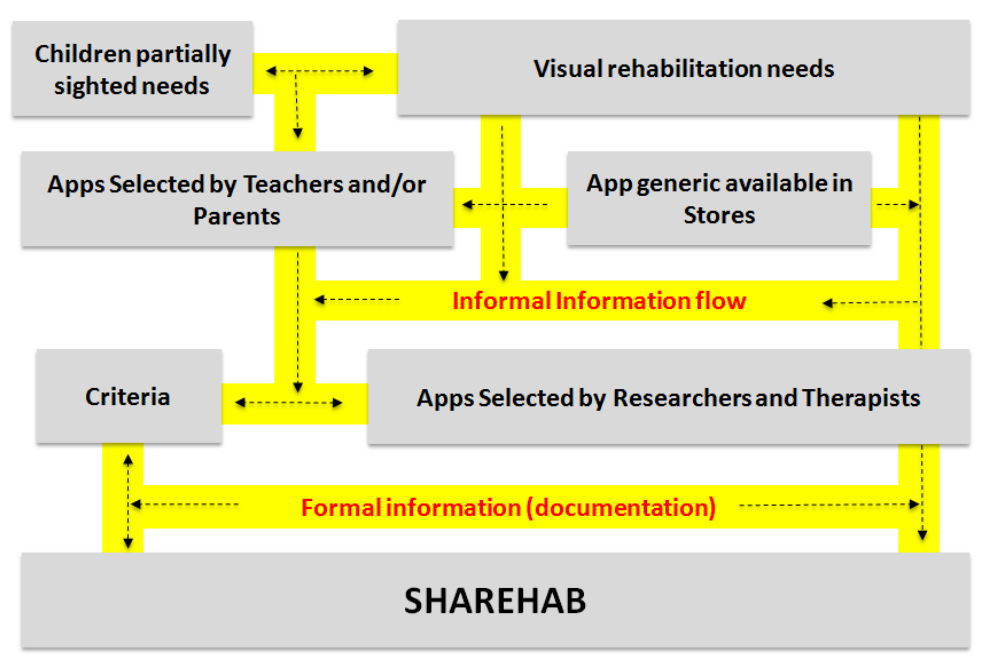

Fig. 1 From apps to Sharehab: the information sharing process 


\section{The Path to Sharehab}

Defining the methodologies and tools adopted in the Sharehab initiative per se is a process that has involved professionals from various fields and backgrounds. The same varied workgroup has also been involved in suggesting the technical specifications and contents of the Sharehab online environment. Project development took place over four phases:

Phase 1: Investigation and definition of the characteristics (graphics and usability) that render apps suitable for visually impaired users, e.g. neutral backgrounds that minimise visual interference with perception of foreground figures and text, simple illustrations with minimal details and bold outlines, etc.;

Phase 2: Empirical validation of these criteria in tests with actual uses in the field;

Phase 3: Analysis of initial candidate apps to be catalogued in Sharehab on condition they met the validated criteria;

Phase 4: Design and implementation of the online platform for information and opinion sharing (platform and database).

From the research viewpoint, the crucial aspect of the project was identifying the criteria that render apps usable for the rehabilitation, education and entertainment of children with visual impairment (Phase 1).

Building on past work in the usability of digital resources generally (Bocconi et al., 2006), the research team concentrated on more recent technology, namely mobile apps. The aim was to identify key elements that underpin app usability on the part of visual impaired children.

As well as surveying apps developed specifically with visually impaired users in mind, the team also investigated apps of a more general nature that therapists and parents had adopted effectively for use by and with visually impaired children. Employment of apps in this latter category had often been made possible thanks to guidance from the research team via different channels, and with the application of suitable adjustments (Dini et al., 2007; Bocconi et al., 2006; 2007).

The app surveying phase highlighted a core set of critical technical criteria in apps (e.g. use of colours, shadings, backgrounds, etc.) that have a strong impact on usability by visually impaired children. Other aspects were identified that, while not directly effecting low vision usability levels, were nonetheless of interest to rehab professionals and parents, such as functions for personalizing the app, changing the interaction language, etc. These characteristics are detailed in Panesi et al. (2018). 
In Phase 2, the research team closely consulted with the therapists, who possess strong practical experience in low vision rehabilitation. Together the group identified an initial set of 200 apps that are actually usable for these ends. For each app, the therapists proposed possible applications in accordance with the type of visual impairment in question.

Phase 3 involved the design and development of a database to store and manage the data emerging from the experimental and field activities, which would subsequently be made available via the specifically designed Sharehab platform. The catalogue entries describing each app are brief and concise, displaying only a subset of the evaluation data and characteristics collected for each individual app.

Finally, Phase 4 involved the entire project team in the design and implementation of a hybrid online environment. This was intended both to make available the collected information destined for rehabilitation purposes and as a means for sharing the information, knowledge, and evaluations generated by the experience of the active user community.

\section{The Online Environment for formal and informal information sharing}

Sharehab can be considered as a hybrid environment, in that it blends documentation, catalogue information, and opinions with the aim of facilitating the identification of resources for low vision rehabilitation. The platform has been optimized for use on mobile devices and provides user-friendly search functions for locating apps to meet the user's specific needs. Only a small number of search criteria are made available: app title, target user age, degree of eyestrain correlated to visual impairment category. This design choice is a deliberate attempt to facilitate use by non-specialist users, such as parents. When the user performs a search, a list of records (under the heading "Occhio alle app!") is generated in response to the search criteria that the user has applied. Filtering functions are available to narrow the list of records so that they align more closely to user interests and requirements. Each individual record on the list comprises an illustrative icon of the app, the app title, a very brief description (in Italian), and a community rating score; to display the complete catalogue entry for the chosen app, the user simply needs to click on the app record title.

The full catalogue entry provides a set of basic information that has been validated by Sharehab researchers. These describe the app and the main interaction features that may impact on usability. There are also indications from therapists that determine what type of visual impairment the app can be used with; this information is the fruit of extensive field tests carried out together with children who have different kinds of visual impairment. 
Users who have registered and logged into Sharehab can also add comments and ratings about the listed apps. Further specialized, technical information regarding rehabilitation use is displayed to rehab professional users only.

As explained, the Sharehab catalogue entry for each app specifically addresses visual impairment matters. However, this specialized information is complemented by more general educational information directly available from the corresponding catalogue entry in Essediquadro $^{2}$, a general-purpose digital educational resources information service also run by CNR-ITD. The corresponding entries in the two services are interlinked to facilitate navigation and to promote a more inclusive approach to the adoption of mobile apps.

Sharehab also features subsections that showcase apps which have caught the attention of the community and/or have attracted favourable comments or ratings. The platform also includes supplementary information pages.

\section{Sharehab's target users}

Sharehab mainly addresses the needs of families of children with visual impairment, rehabilitation professionals and teachers. The platform offers users three different access levels (Guest, Basic and Pro) for accessing contents and contributing to the knowledge building process.

Anyone can explore Sharehab as a Guest user, so as to get a taste of the platform and the project itself, and therefore decide whether or not to join the community. Guest users can consult listings of a variety of apps suitable for children with visual impairment and access basic information about the criteria guiding app choice.

At the other end of the scale, rehab professionals, orthoptists and visual rehabilitation therapists can register as Pro users. This grants them access to supplementary technical information regarding particular apps for rehabilitation. The added value at this level lies in the opportunity for professional peers to exchange of know-how expressed in familiar technical, sector-specific language. This regards aspects like the application of technical aids, compatibility with accessibility features, management of involuntary user movement, ergonomics, and experience-based knowledge of target user needs. To identify which apps are best suited to particular rehab needs, Pro users can search the catalogue using a specific menu of general sight function categories. Each function is related to a specific rehabilitation methodology and objective.

\footnotetext{
2 https://sd2.itd.cnr.it/
} 
The bulk of Sharehab users register in the Basic category, which effectively represents the core of the Sharehab community. This largely comprises sector professionals, teachers and parents. Basic users can share their experiences and opinions of the apps listed in Sharehab, and can also propose new apps to be listed on the platform based on their practical and/or professional experience. Participation in the community is open to all Basic users who are willing to share their experience and expertise and to enrich the community's knowledge base with their own information, whether of a professional nature or not.

It was decided not to add a general discussion space on the platform, as this could well have ended up being dominated by a small number of highly active, experienced members, possibly discouraging others from sharing their experience. Conversely, a more discreet form of participation was foreseen, whereby users can post comments tied to specific product listings. In this way, the primary focus for user feedback is on the app and on their experience and impressions thereof.

As is the case in most content-centred educational communities (Trentin, 2004), interactions in Sharehab affect the dynamics surrounding the content. Typically, the opinions, ratings, and displayed pages tend to centre around the sections of the platform that highlight the most commonly viewed and highly rated apps, those that have attracted the most recent comments, and the "Top Apps" (combined rating and comment level).

Such features help to provide stimulus and focal points for interaction, especially for new users, without limiting the scope for freedom of expression. There is ample space for sharing experiences, and for informing others about salient characteristics to consider when choosing a usable product that meets specific needs.

The consolidation and growth of Sharehab is grounded in its involving and expanding user community. This comes not just through uses sharing opinions and ratings about the apps already listed on the platform, but also by allowing them to propose inclusion of new apps that they themselves have used effectively in their practice and activities. When the Sharehab editorial team evaluates these and shares them on the platform, a virtuous circle of knowledge sharing and knowledge building is generated that is the essential drive force of the Sharehab initiative and the key to its sustainability.

Added value derives from ongoing interaction and exchange between people who share a common objective, in this case support for children with visual impairment. Without Sharehab, this value would be lost to those who could benefit from it most. 


\section{Conclusion}

Given their high degree of manageability, mobile devices like smartphones and tablets now form part of the daily lives of most children and youngsters, including those with visual impairment. They use tablets in particular in a range of different settings: for home entertainment, for learning at school, and also for carrying out rehabilitation exercises.

Given the enormous number of apps available on the market, making a suitable choice for low vision users can be a complex matter. Accordingly, the need arises to identify and apply criteria for selecting apps that propose activities and contents that align appropriately with the end user's age group and, at the same time, provide insight into the characteristics unpinning contents, interface and interactions.

One way to address the challenge of identifying such apps is to rely on authoritative, institutional online cataloguing and documentation services that seek to provide information in a neutral and objective manner. Another is to seek out the experience and opinion of others who have actually had practical experience in using such apps and hence are aware of their potential and limitations.

This latter type of information typically flows in an informal and spontaneous manner within social networking contexts. This form of communication exchange is increasingly becoming part of the research world too as an amplifier underpinning knowledge building processes.

It is our firm belief that children with visual impairment can and should be able to use emerging technologies like apps, so long as these respond to certain requirements. This can be achieved by channelling the informal information spontaneously generated and exchanged online by users within structured documentation services. This is the hybrid approach pursued by Sharehab, which combines formal and informal information in a manner that leaves the way open for dynamic ongoing enrichment. Sharehab's challenge is to respond successfully to the diverse needs of different actors and stakeholders, such as therapists, teachers and parents, who are seeking to select appropriate apps for children with low vision that can then be used in a range of settings and for different purposes, whether it be rehabilitation, learning or entertainment. On a final note, we believe that the Sharehab model could be successfully adopted to meet the needs of other kinds of disability in addition to visual impairment. 


\section{Acknowledgments}

Sharehab was developed as part of a project financed by Fondazione Vodafone Italia as part of their "Digital for Social 2015" initiative. We wish to thank all those who were involved in the project and its activities, especially the children with low vision attending the Chiossone Institute's Centre for Visual Rehabilitation, and their parents.

\section{References}

Bocconi, S., Dini, S., Ferlino, L. \& Martinoli, C. (2007). ICT Educational Tools and Visually Impaired Students:Different Answers to Different Accessibility Needs. Lecture Notes in Computer Science, vol. 4556 (7); p. 491-500

Bocconi, S., Dini, S., Ferlino, L. \& Ott, M. (2006). Accessibility of educational multimedia: in search of specific standards. International Journal of Emerging Technologies in Learning, 1(3), 1-5

Bonaiuti G., Calvani A., Menichetti L., Vivanet G. (2017). Le tecnologie educative, Roma: Carocci editore

Dini, S., Ferlino, L. \& Martinoli, C. (2004) Usability of educational software for visual impairment: a question of viewpoint, in Computer Helping People with special needs, 9th International conference ICCHP Paris (France): 575-582

Dini, S., Ferlino, L. (2016), La conoscenza tra le dita dei bambini. Imparare e giocare a tempo di app, TD Tecnologie Didattiche, 24 (3), 147-155

Panesi, S., Caruso, G.P, Ferlino, L., Dini, S. (2018). Choosing apps for activities for rehabilitation, learning, and leisure activities involving visually-impaired children. Proceedings of the EDULEARN 10th International Conference on Education and New Learning Technologies, Palma, Spain. 2-4 July.

Pantazopoulos, G.A. (2017). Knowledge Networks: A Key Driver for Technological Advancement and Social Progress. Journal of Failure Analysis and Prevention, 17 (5) pp 823-824|

Trentin, G., (2004). Apprendimento in rete e condivisione delle conoscenze. Milano: Franco Angeli 